\title{
Metallicity estimates for old star clusters in M 33
}

\author{
Jun Ma, Xu Zhou, and Jiansheng Chen \\ National Astronomical Observatories, Chinese Academy of Sciences, Beijing 100012, PR China \\ Received 18 February 2003 / Accepted 7 August 2003

\begin{abstract}
Using the theoretical stellar population synthesis models of BC96, Kong et al. (2003) showed that some BATC colors and color indices could be used to disentangle the age and metallicity effect. They found that there is a very good relation between the flux ratio of $L_{8510} / L_{9170}$ and the metallicity for stellar populations older than $1 \mathrm{Gyr}$. In this paper, based on the Kong et al. results and on the multicolor spectrophotometry of Ma et al. (2001, 2002a,b,c), we estimate the metallicities of 31 old star clusters in the nearby spiral galaxy M 33, 23 of which are "true" globular clusters. The results show that most of these old clusters are metal poor. We also find that the ages and metal abundance for these old star clusters of M 33 do not vary with
\end{abstract} \\ deprojected radial position.
}

Key words. galaxies: individual: M 33 - galaxies: evolution - galaxies: star clusters

\section{Introduction}

Globular clusters (GCs) are thought to be among the oldest radiant objects in the Universe. They are simple coeval stellar systems which formed on a very short timescale during phases of intense star formation in host galaxies. The GCs of the Milky Way probe the manner in which our Galaxy formed. Studies of similar populations in other galaxies can reveal the properties of these galaxies soon after their formation. For example, the widely varying specific frequency of GCs in individual galaxies indicates that cluster formation is almost certainly affected by the local environment with the host galaxy.

M 33, at a distance of $850 \mathrm{kpc}$, is the third-brightest member of the Local Group, and is classified as a late-type ScII-III spiral (1999). This galaxy represents a morphological type intermediate between the largest "early-type" spirals and the dwarf irregulars in the Local Group (Chandar et al. 1999a). M 33 subtends $1^{\circ}$ on the sky. Its large angular extent and favorable inclination $i=56^{\circ}$ (Regan \& Vogel 1994) make it suitable for studies of stellar content. However, its large size makes its study difficult. The Beijing-Arizona-TaiwanConnecticut (BATC) Multicolor Sky Survey (Fan et al. 1997; Zheng et al. 1999) obseves M 33 as part of its galaxy calibration program.

Before Chandar et al. (1999a,b,c, 2001, 2002), the system of clusters in M 33 were not well studied, although detection, and photometry and spectrophotometry have been obtained (see details from Hiltner 1960; Kron \& Mayall 1960; Melnick \& D'Odorico 1978; Christian \& Schommer 1982, 1988; Huchra et al. 1996). Now, a database of about 400 star

Send offprint requests to: Jun Ma, e-mail: majun@vega.bac.pku.edu.cn clusters is available from the ground-based work, and from the Hubble Space Telescope (HST) images.

Using the theoretical stellar population synthesis models of Bruzual \& Charlot (1996, unpublished, hereafter BC96) and multicolor photometry, Kong et al. (2000) studied the age, metallicity, and interstellar-medium reddening distribution for M 81. When they convolved the spectral energy distributions (SEDs) of BC96 with the BATC filter profiles to obtain the optical and near-integrated luminosity, they found that, among all the BATC filter bands, the color index centered at $8510 \AA$ is much more sensitive to the metallicity than to the age. The center of this filter band is near the Ca II triplets $(\lambda \lambda=$ 8498, 8542, 8662 Å) (hereafter CaT). As Zhou (1991) noted, that the strength of the CaT depends on the effective temperature, surface gravity, and the metallicity in late-type stars. A very good relation between the flux ratio of $I_{8510} \equiv L_{8510} / L_{9170}$ and the metallicity was found for stellar populations older than 1 Gyr in Kong et al. (2000).

In this paper, we estimate the metallicities of the 31 old star clusters that were detected by Christian \& Schommer (1982), Chandar et al. (1999a, 2001), and by Mochejska et al. (1998) in M 33 using the relation of Kong et al. (2000). The ages of all these sample star clusters were estimated by Ma et al. (2001, 2002a,b,c) by comparing the BC96 simple stellar population synthesis models with the integrated photometric measurements.

The outline of the paper is as follows. Sample selection, observations and data reduction are given in Sect. 2. Section 3 presents the spectral synthesis models. In Sect. 4, we provide a brief description of the method of Kong et al. (2000), and estimate the metallicities of 31 sample old star clusters. Some statistical properties of old star clusters are investigated in Sect. 5. In Sect. 6, we give our major results and some discussions. 


\section{Sample of star clusters, observations and data reduction}

\subsection{Sample of old star clusters}

The sample of star clusters in this paper is from Ma et al. (2001, 2002a,b,c), who presented multicolor photometry and estimated the ages using BC96 models for 180 star clusters in M 33. The RA and Dec of these clusters are from Christian \& Schommer (1982), Chandar et al. (1999a, 2001), or Mochejska et al. (1998). Christian \& Schommer (1982) detected more than 250 nonstellar objects using $14 \times 14$ inch $^{2}$ unfiltered, unbaked, IIa-O focus plates exposed for 150 minutes with the Kitt Peak 4 m Richey-Chrétien (R-C) direct camera. Chandar et al. (1999a, 2001) used 55 multiband HST WFPC2 fields to search for star clusters much closer to the nucleus of M 33 than previous studies, and detected 162 star clusters, 131 of which were previously unknown. Mochejska et al. (1998) detected 51 globular cluster candidates in M 33, 32 of which were not previously cataloged, using the data collected in the DIRECT project (1998; 1998). Ma et al. (2001, 2002a,b,c) obtained the SEDs of the 180 clusters by aperture photometry, and estimated their ages using the theoretical evolutionary population synthesis methods. In Ma et al. (2001), there are 10 clusters, the ages of which are older than 1 Gyr. We exclude three star clusters of these 10 because of their low signal-to-noise ratios. Also, cluster 54 in Chandar et al. (1999a) is U137 in Christian \& Schommer (1982). In Ma et al. (2002b), there are 22 clusters older than 1 Gyr. However, the ratios of signal-to-noise of 11 clusters are not high enough, and are not included in this sample. In Ma et al. (2002c), there are 5 clusters older than 1 Gyr. The signal-to-noise ratio of cluster 2 is not high enough, and is also not included in this sample. Altogether, there are 31 old star clusters in this paper. Figure 1 is the image of M 33 in filter BATC07 (5785 $\AA$ ), the circles indicate the positions of the sample clusters. By comparing the photometric measurements to integrated colors from theoretical models by Bertelli et al. (1994), Chandar et al. (1999b, 2002) estimated ages for 23 old star clusters in common. Table 1 lists the comparison of age estimates with previously results (Chandar et al. 1999b, 2002). Except for clusters 49 and 59 of Chandar et al. (1999a), the ages estimated by Ma et al. (2001, 2002a,b,c) are consistent with the ones estimated by Chandar et al. (1999b, 2002). Cluster 49, the $B-V$ value of which is very large 0.824 (Chandar et al. 1999b), should be an old cluster. Our sample includes 23 "true" globular clusters, which have $(B-V)_{0} \geq 0.6$ or $(V-I)_{0} \geq 0.78$, colors typical of Galactic GCs (Chandar et al. 2001).

\subsection{Observations and data reduction}

The large field multicolor observations of the spiral galaxy M 33 were collected using the Ford Aerospace $2048 \times$ 2048 CCD mosaic camera on the $60 / 90 \mathrm{~cm} \mathrm{f} / 3$ Schmidt telescope of the Xinglong station of the National Astronomical Observatories. The field of view of the CCD is $58^{\prime} \times 58^{\prime}$ with a pixel scale of $1^{\prime}$.7. The typical seeing of the Xinglong station is $2^{\prime \prime}$. The multicolor BATC filter system, which was specifically designed to avoid contamination from the

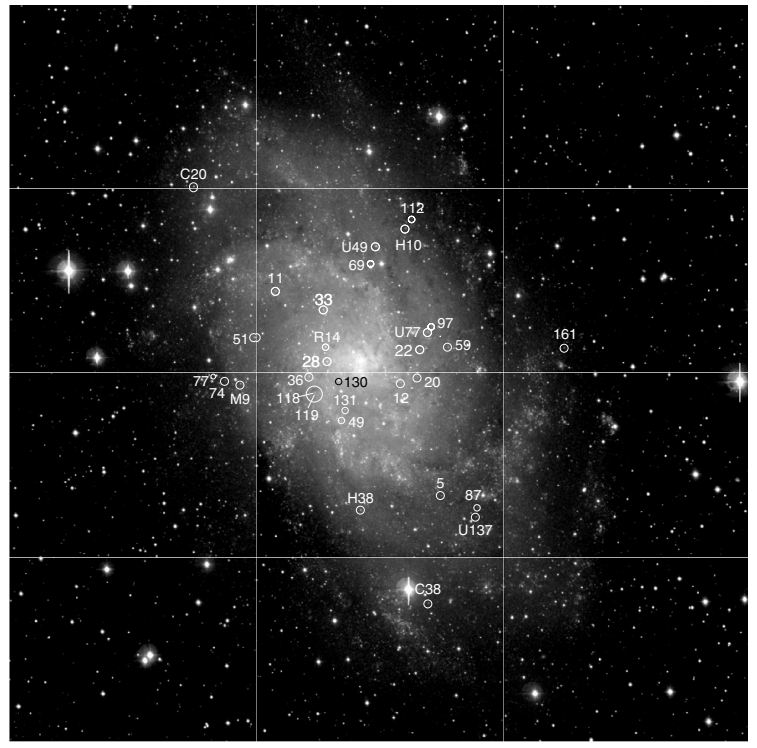

Fig. 1. The image of M 33 in filter BATC07 (5785 A) and the positions of the sample star clusters. The center of the image is located at $\mathrm{RA}=01^{\mathrm{h}} 33^{\mathrm{m}} 50.58 \mathrm{Dec}=30^{\circ} 39^{\prime} 08^{\prime \prime} 4(\mathrm{~J} 2000.0)$. North is up and east is to the left.

brightest and most variable night sky emission lines, includes 15 intermediate-band filters, covering the total optical wavelength range from 3000 to $10000 \AA$. It is defined the magnitude zero points similar to the spectrophotometric $\mathrm{AB}$ magnitude system, a $\tilde{f}_{v}$ monochromatic system (Oke \& Gunn 1983) based on the SEDs of the four $F$ sub-dwarfs, HD 19445, $\mathrm{HD} 84937, \mathrm{BD}+26^{\circ} 2606$, and $\mathrm{BD}+17^{\circ} 4708$. The advantage of the $\mathrm{AB}$ magnitude system is that the magnitude is directly related to physical units. The BATC magnitude system is defined as the AB magnitude system,

$m_{\text {batc }}=-2.5 \log \tilde{F}_{v}-48.60$,

where $\tilde{F}_{v}$ is the appropriately averaged monochromatic flux in units of erg s${ }^{-1} \mathrm{~cm}^{-2} \mathrm{~Hz}^{-1}$ at the effective wavelength of the specific passband. In the BATC system (Yan et al. 2000), $\tilde{F}_{v}$ is defined as

$\tilde{F}_{v}=\frac{\int \mathrm{d}(\log v) f_{v} R_{v}}{\int \mathrm{d}(\log v) R_{v}}$,

which links the magnitude to the number of photons detected by the CCD rather than to the input flux (Fukugita et al. 1996). In this equation, $R_{v}$ is the system response, $f_{v}$ is the SED of the source.

The images of M 33 covering the whole optical body of M 33 were accumulated in 13 intermediate band filters with a total exposure time of about 37.25 hours from September 23, 1995 to August 28, 2000. Data reduction, by bias subtraction and flat-fielding with dome flats, was performed with the automatic data reduction software PIPELINE I developed for the BATC multicolor sky survey $(1996 ; 1999)$. The dome flat-field images were taken by using a diffuse plate in front of the correcting plate of the Schmidt telescope. We performed photometric calibration of the M 33 images using the Oke-Gunn primary flux standard stars HD 19445, HD 84937, BD +26²606, 
Table 1. Comparion of age estimates with previous measurements.

\begin{tabular}{ccc}
\hline \hline Cluster $^{\mathrm{a}}$ & $\begin{array}{c}\text { Chandar et al. } \\
\text { log age (yr) }\end{array}$ & $\begin{array}{c}\text { Ma et al. } \\
\text { log age (yr) }\end{array}$ \\
\hline U 49 & $9.2 \pm 0.1$ & 9.60 \\
R 12 & $9.7 \pm 0.1$ & 10.00 \\
R 14 & $10.2 \pm 0.2$ & 9.11 \\
M 9 & $9.2 \pm 0.1$ & 9.63 \\
U 77 & $9.15 \pm 0.15$ & 9.20 \\
H 38 & $9.25 \pm 0.15$ & 9.70 \\
C 20 & $9.2 \pm 0.1$ & 9.95 \\
C38 & $8.9 \pm 0.1$ & 9.28 \\
H 10 & $9.25 \pm 0.15$ & 9.90 \\
U 137 & $9.35 \pm 0.15$ & 10.27 \\
CBF 11 & $9.50 \pm 0.30$ & 10.30 \\
CBF 20 & $10.1 \pm 0.20$ & 9.54 \\
CBF 22 & $9.25 \pm 0.15$ & 9.26 \\
CBF 28 & $10.2 \pm 0.40$ & 9.80 \\
CBF 49 & $7.90 \pm 0.20$ & 9.34 \\
CBF 59 & $7.40 \pm 0.20$ & 9.11 \\
CBF 69 & $9.2 \pm 0.1$ & 9.76 \\
CBF 74 & $9.3 \pm 0.1$ & 9.32 \\
CBF 97 & $9.3 \pm 0.2$ & 10.28 \\
CBF 112 & $8.8 \pm 0.2$ & 9.21 \\
CBF 118 & $9.15 \pm 0.15$ & 9.16 \\
CBF 161 & $9.1 \pm 0.1$ & 9.23 \\
M 12 & $9.4 \pm 0.2$ & 9.63 \\
\hline
\end{tabular}

${ }^{a}$ CBF identifications are from Chandar et al. (1999a, 2001); M identifications are from Mochejska et al. (1998); The others are from Christian \& Schommer (1982).

and $\mathrm{BD}+17^{\circ} 4708$, which were observed during photometric nights (see details from Yan et al. 2000; Zhou et al. 2001).

Using the images of the standard stars observed on photometric nights, we derive iteratively the extinction curves and the slight variation of the extinction coefficients with time (Zhou et al. 2001). The extinction coefficients at any given time in a night $[K+\Delta K(U T)]$ and the zero point of the instrumental magnitude $(C)$ were obtained by

$m_{\text {batc }}=m_{\text {inst }}+[K+\Delta K(U T)] X+C$,

where $X$ is air mass. The instrumental magnitudes $\left(m_{\text {inst }}\right)$ of the selected bright, isolated and unsaturated stars on the M 33 images of the same photometric nights can be readily transformed to the BATC AB magnitude system $\left(m_{\text {batc }}\right)$. We calibrated the photometry on the combined images by comparing the magnitudes of these stars to determine a mean magnitude offset to the photometric images. Table 2 lists the parameters of the BATC filters and the statistics of observations. Column 6 of Table 2 gives the zero point error, in magnitude, for the standard stars in each filter. The formal errors we obtain for these stars in the 13 BATC filters are $\$ 0.02 \mathrm{mag}$. This indicates that we can define the standard BATC system to an accuracy of $\lesssim 0.02$ mag.

\subsection{Integrated photometry}

For each star cluster, aperture photometry was used to obtain magnitudes. To avoid contamination from nearby objects, we
Table 2. Parameters of the BATC filters and statistics of observations.

\begin{tabular}{cccccc}
\hline \hline No. & Name & cw $^{\mathrm{a}}(\AA)$ & Exp. (hr) & N.img $^{\mathrm{b}}$ & $\mathrm{rms}^{\mathrm{c}}$ \\
\hline 1 & BATC03 & 4210 & $00: 55$ & 04 & 0.024 \\
2 & BATC04 & 4546 & $01: 05$ & 04 & 0.023 \\
3 & BATC05 & 4872 & $03: 55$ & 19 & 0.017 \\
4 & BATC06 & 5250 & $03: 19$ & 15 & 0.006 \\
5 & BATC07 & 5785 & $04: 38$ & 17 & 0.011 \\
6 & BATC08 & 6075 & $01: 26$ & 08 & 0.016 \\
7 & BATC09 & 6710 & $01: 09$ & 08 & 0.006 \\
8 & BATC10 & 7010 & $01: 41$ & 08 & 0.005 \\
9 & BATC11 & 7530 & $02: 07$ & 10 & 0.017 \\
10 & BATC12 & 8000 & $03: 00$ & 11 & 0.003 \\
11 & BATC13 & 8510 & $03: 15$ & 11 & 0.005 \\
12 & BATC14 & 9170 & $04: 45$ & 25 & 0.011 \\
13 & BATC15 & 9720 & $05: 00$ & 26 & 0.009 \\
\hline
\end{tabular}

${ }^{\text {a }}$ Central wavelength for each BATC filter.

${ }^{\mathrm{b}}$ Image numbers for each BATC filter.

${ }^{c}$ Zero point error, in magnitude, for each filter as obtained from the standard stars.

adopt a small aperture of $6^{\prime \prime} .8$ corresponding to a diameter of 4 pixels in the Ford CCD. The large-aperture measures on the uncrowded bright stars were used to determine the aperture corrections, i.e., the magnitude difference between the smallaperture magnitude and the "total" or seeing-independent magnitude for the stars on each frame. The uncertainties for each filter take into account the error from the object count rate, sky variance, and instrument gain. For convenience, we present Table 3 with multiband photometry for sample clusters in this paper based on Ma et al. (2001, 2002a,b,c). Column 1 is cluster number. Column 2 to Col. 14 show the magnitudes of different bands. The second line of each star cluster is the uncertainties of magnitude of corresponding band.

\section{Spectral synthesis}

Since the pioneering works of Tinsley (1972) and Searle et al. (1973), spectral population synthesis has become a standard technique to study the stellar populations of galaxies. Since stellar clusters can be assumed single age and single metallicity group of stars, their integrated colors reflect their age and metallicity for a given initial mass function. A comprehensive compilation of various evolutionary synthesis models was presented by Leitherer et al. (1996) and Kennicutt (1998). One of the widely used models is BC96. In this model, the evolution of the spectrophotometric properties for a wide range of stellar metallicity, $Z=0.0004,0.004,0.008,0.02,0.05$, and 0.1 , are presented. The evolving spectra include the contribution of the stellar component in the range from the EUV to the FIR. The age varies from 0 to $20 \mathrm{Gyr}$ and various IMFs are considered.

To estimate the ages of star clusters in M 33, we convolve the SED of BC96 with BATC filter profiles to obtain the 
Table 3. SEDs of 31 old star clusters.

\begin{tabular}{|c|c|c|c|c|c|c|c|c|c|c|c|c|c|}
\hline $\begin{array}{c}\text { Cluster }^{\mathrm{a}} \\
\text { (1) }\end{array}$ & $\begin{array}{l}03 \\
(2)\end{array}$ & $\begin{array}{l}04 \\
\text { (3) }\end{array}$ & $\begin{array}{l}05 \\
(4)\end{array}$ & $\begin{array}{l}06 \\
(5)\end{array}$ & $\begin{array}{l}07 \\
\text { (6) }\end{array}$ & $\begin{array}{l}08 \\
\text { (7) }\end{array}$ & $\begin{array}{l}09 \\
(8)\end{array}$ & $\begin{array}{l}10 \\
(9)\end{array}$ & $\begin{array}{c}11 \\
(10)\end{array}$ & $\begin{array}{c}12 \\
(11)\end{array}$ & $\begin{array}{c}13 \\
(12)\end{array}$ & $\begin{array}{c}14 \\
(13)\end{array}$ & $\begin{array}{c}15 \\
(14)\end{array}$ \\
\hline \multirow[t]{2}{*}{ U 49 } & 16.99 & 16.56 & 16.46 & 16.27 & 16.06 & 15.98 & 15.82 & 15.78 & 15.70 & 15.58 & 15.52 & 15.48 & 15.40 \\
\hline & 0.022 & 0.018 & 0.014 & 0.015 & 0.010 & 0.011 & 0.011 & 0.010 & 0.010 & 0.009 & 0.010 & 0.012 & 0.012 \\
\hline \multirow[t]{2}{*}{ R 12} & 17.34 & 16.81 & 16.62 & 16.43 & 16.15 & 16.11 & 15.90 & 15.83 & 15.77 & 15.68 & 15.56 & 15.51 & 15.44 \\
\hline & 0.049 & 0.036 & 0.027 & 0.029 & 0.020 & 0.021 & 0.022 & 0.023 & 0.022 & 0.019 & 0.021 & 0.020 & 0.024 \\
\hline \multirow[t]{2}{*}{ R 14} & 17.55 & 17.04 & 16.84 & 16.59 & 16.21 & 16.14 & 15.91 & 15.81 & 15.66 & 15.55 & 15.43 & 15.32 & 15.20 \\
\hline & 0.052 & 0.037 & 0.027 & 0.028 & 0.019 & 0.021 & 0.017 & 0.018 & 0.020 & 0.016 & 0.017 & 0.017 & 0.021 \\
\hline \multirow[t]{2}{*}{ M9 } & 17.82 & 17.48 & 17.38 & 17.17 & 16.96 & 16.90 & 16.73 & 16.68 & 16.60 & 16.53 & 16.45 & 16.39 & 16.38 \\
\hline & 0.046 & 0.034 & 0.025 & 0.026 & 0.016 & 0.018 & 0.016 & 0.018 & 0.018 & 0.017 & 0.025 & 0.021 & 0.026 \\
\hline \multirow[t]{2}{*}{ U 77} & 17.94 & 17.51 & 17.38 & 17.20 & 17.05 & 16.97 & 16.77 & 16.75 & 16.68 & 16.59 & 16.58 & 16.49 & 16.41 \\
\hline & 0.066 & 0.047 & 0.042 & 0.039 & 0.025 & 0.024 & 0.033 & 0.024 & 0.030 & 0.028 & 0.030 & 0.031 & 0.044 \\
\hline \multirow[t]{2}{*}{ H 38} & 18.03 & 17.68 & 17.52 & 17.27 & 17.06 & 16.99 & 16.80 & 16.71 & 16.69 & 16.61 & 16.55 & 16.46 & 16.49 \\
\hline & 0.035 & 0.028 & 0.021 & 0.020 & 0.016 & 0.016 & 0.015 & 0.015 & 0.016 & 0.015 & 0.022 & 0.019 & 0.026 \\
\hline \multirow[t]{2}{*}{ C 20} & 18.44 & 18.05 & 17.87 & 17.70 & 17.50 & 17.42 & 17.24 & 17.26 & 17.13 & 17.00 & 16.90 & 16.84 & 16.81 \\
\hline & 0.037 & 0.026 & 0.022 & 0.022 & 0.017 & 0.020 & 0.017 & 0.020 & 0.019 & 0.019 & 0.025 & 0.021 & 0.039 \\
\hline \multirow[t]{2}{*}{ C 38} & 18.71 & 18.35 & 18.28 & 18.05 & 17.98 & 17.88 & 17.78 & 17.70 & 17.73 & 17.73 & 17.80 & 17.80 & 17.72 \\
\hline & 0.080 & 0.050 & 0.040 & 0.058 & 0.029 & 0.040 & 0.032 & 0.037 & 0.034 & 0.041 & 0.058 & 0.061 & 0.089 \\
\hline \multirow[t]{2}{*}{ H 10} & 19.27 & 18.73 & 18.54 & 18.21 & 17.96 & 17.76 & 17.53 & 17.51 & 17.31 & 17.21 & 17.19 & 17.10 & 17.03 \\
\hline & 0.093 & 0.055 & 0.043 & 0.038 & 0.030 & 0.030 & 0.033 & 0.031 & 0.029 & 0.029 & 0.033 & 0.036 & 0.053 \\
\hline \multirow[t]{2}{*}{ U 137} & 19.21 & 18.88 & 18.68 & 18.42 & 18.22 & 18.10 & 17.88 & 17.80 & 17.81 & 17.74 & 17.71 & 17.54 & 17.61 \\
\hline & 0.082 & 0.058 & 0.043 & 0.043 & 0.030 & 0.030 & 0.029 & 0.031 & 0.033 & 0.036 & 0.049 & 0.042 & 0.065 \\
\hline \multirow[t]{2}{*}{ CBF 11} & 19.56 & 19.05 & 18.99 & 18.82 & 18.50 & 18.52 & 18.19 & 18.11 & 17.99 & 17.95 & 17.67 & 17.62 & 17.54 \\
\hline & 0.153 & 0.098 & 0.080 & 0.085 & 0.063 & 0.075 & 0.052 & 0.053 & 0.050 & 0.067 & 0.081 & 0.046 & 0.071 \\
\hline \multirow[t]{2}{*}{ CBF 20} & 19.11 & 18.76 & 18.70 & 18.45 & 18.31 & 18.25 & 18.05 & 17.83 & 17.70 & 17.71 & 17.52 & 17.39 & 17.30 \\
\hline & 0.102 & 0.077 & 0.063 & 0.063 & 0.054 & 0.061 & 0.048 & 0.043 & 0.040 & 0.055 & 0.072 & 0.038 & 0.058 \\
\hline \multirow[t]{2}{*}{ CBF 22} & 18.66 & 18.23 & 18.13 & 18.01 & 17.76 & 17.79 & 17.74 & 17.63 & 17.60 & 17.49 & 17.56 & 17.49 & 17.32 \\
\hline & 0.065 & 0.046 & 0.037 & 0.040 & 0.032 & 0.038 & 0.035 & 0.034 & 0.035 & 0.043 & 0.071 & 0.040 & 0.056 \\
\hline \multirow[t]{2}{*}{ CBF 28} & 17.26 & 16.75 & 16.60 & 16.46 & 16.16 & 16.15 & 15.97 & 15.91 & 15.87 & 15.78 & 15.72 & 15.69 & 15.66 \\
\hline & 0.024 & 0.017 & 0.013 & 0.014 & 0.010 & 0.012 & 0.010 & 0.010 & 0.010 & 0.011 & 0.015 & 0.010 & 0.014 \\
\hline \multirow[t]{2}{*}{ CBF 49} & 19.37 & 18.54 & 18.56 & 18.52 & 18.08 & 18.07 & 17.86 & 17.91 & 17.93 & 17.74 & 17.80 & 17.81 & 17.75 \\
\hline & 0.151 & 0.073 & 0.065 & 0.078 & 0.049 & 0.057 & 0.046 & 0.050 & 0.054 & 0.059 & 0.094 & 0.059 & 0.090 \\
\hline \multirow[t]{2}{*}{ CBF 59} & 18.47 & 18.29 & 18.28 & 18.19 & 17.92 & 17.98 & 17.87 & 17.69 & 17.48 & 17.38 & 17.34 & 17.16 & 17.05 \\
\hline & 0.046 & 0.038 & 0.034 & 0.037 & 0.033 & 0.036 & 0.031 & 0.029 & 0.026 & 0.034 & 0.050 & 0.028 & 0.041 \\
\hline \multirow[t]{2}{*}{ CBF 69} & 19.36 & 19.10 & 18.99 & 18.53 & 18.62 & 18.48 & 18.34 & 18.28 & 18.19 & 18.21 & 17.96 & 17.90 & 18.08 \\
\hline & 0.154 & 0.125 & 0.135 & 0.155 & 0.100 & 0.088 & 0.093 & 0.087 & 0.099 & 0.103 & 0.106 & 0.102 & 0.163 \\
\hline \multirow[t]{2}{*}{ CBF 74} & 19.93 & 19.43 & 19.14 & 18.87 & 18.68 & 18.59 & 18.47 & 18.40 & 18.41 & 18.16 & 18.28 & 18.09 & 18.08 \\
\hline & 0.201 & 0.142 & 0.095 & 0.100 & 0.064 & 0.069 & 0.064 & 0.068 & 0.072 & 0.061 & 0.098 & 0.076 & 0.101 \\
\hline \multirow[t]{2}{*}{ CBF 77} & 18.53 & 18.33 & 18.35 & 18.04 & 18.08 & 17.80 & 17.82 & 17.82 & 17.64 & 17.50 & 17.57 & 17.46 & 17.09 \\
\hline & 0.061 & 0.045 & 0.039 & 0.046 & 0.032 & 0.036 & 0.030 & 0.036 & 0.035 & 0.028 & 0.049 & 0.037 & 0.054 \\
\hline \multirow[t]{2}{*}{ CBF 87} & 19.91 & 19.44 & 19.22 & 18.96 & 18.89 & 18.68 & 18.53 & 18.45 & 18.50 & 18.35 & 18.19 & 18.23 & 18.28 \\
\hline & 0.143 & 0.090 & 0.060 & 0.057 & 0.045 & 0.051 & 0.051 & 0.052 & 0.055 & 0.047 & 0.072 & 0.075 & 0.102 \\
\hline
\end{tabular}

optical and near-infrared integrated luminosity. The integrated luminosity $L_{\lambda_{i}}(t, Z)$ of the $i$ th BATC filter can be calculated as

$L_{\lambda_{i}}(t, Z)=\frac{\int F_{\lambda}(t, Z) \varphi_{i}(\lambda) \mathrm{d} \lambda}{\int \varphi_{i}(\lambda) \mathrm{d} \lambda}$,

where $F_{\lambda}(t, Z)$ is the SED of the BC96 of metallicity $Z$ at age $t$, $\varphi_{i}(\lambda)$ is the response functions of the $i$ th filter of the BATC filter system $(i=3,4, \ldots, 15)$, respectively. To avoid using distancedependent parameters, we calculate the integrated colors of a BC96 relative to the BATC filter BATC08 $(\lambda=6075 \AA)$ :

$$
C_{\lambda_{i}}(t, Z)=L_{\lambda_{i}}(t, Z) / L_{6075}(t, Z)
$$

As a result, we obtain intermediate-band colors for 6 metallicities from $Z=0.0004$ to $Z=0.1$. Then, we determined the ages and best-fit models of metallicity by minimizing the difference between the intrinsic and integrated colors of BC96,

$R^{2}(n, t, Z)=\sum_{i=3}^{15}\left[C_{\lambda_{i}}^{\mathrm{intr}}(n)-C_{\lambda_{i}}^{\mathrm{ssp}}(t, Z)\right]^{2}$,

where $C_{\lambda_{i}}^{\mathrm{ssp}}(t, Z)$ represents the integrated color in the $i$ th filter of a SSP with age $t$ and metallicity $Z$, and $C_{\lambda_{i}}^{\text {intr }}(n)$ is the intrinsic integrated color for nth starcluster. For convenience, we also list the ages of sample star clusters of this paper in Col. 5 of Table 4. The uncertainties in the age estimates arising from photometric uncertainties are 0.2 or so, i.e., age $\pm 0.2 \times$ age $[\log \mathrm{yr}]$, and are formal errors that do not include the model uncertainties. The star cluster ages obtained in this paper are model-dependent and do not represent "absolute values". Uncertainties exist in the stellar evolution, in 
Table 3. continued.

\begin{tabular}{|c|c|c|c|c|c|c|c|c|c|c|c|c|c|}
\hline $\begin{array}{c}\text { Cluster }^{\mathrm{a}} \\
\text { (1) }\end{array}$ & $\begin{array}{l}03 \\
(2)\end{array}$ & $\begin{array}{l}04 \\
(3)\end{array}$ & $\begin{array}{l}05 \\
(4)\end{array}$ & $\begin{array}{l}06 \\
(5)\end{array}$ & $\begin{array}{l}07 \\
(6)\end{array}$ & $\begin{array}{l}08 \\
(7)\end{array}$ & $\begin{array}{l}09 \\
(8)\end{array}$ & $\begin{array}{l}10 \\
(9)\end{array}$ & $\begin{array}{c}11 \\
(10)\end{array}$ & $\begin{array}{c}12 \\
(11)\end{array}$ & $\begin{array}{c}13 \\
(12) \\
\end{array}$ & $\begin{array}{c}14 \\
(13)\end{array}$ & $\begin{array}{c}15 \\
(14)\end{array}$ \\
\hline \multirow[t]{2}{*}{ CBF 87} & 19.91 & 19.44 & 19.22 & 18.96 & 18.89 & 18.68 & 18.53 & 18.45 & 18.50 & 18.35 & 18.19 & 18.23 & 18.28 \\
\hline & 0.143 & 0.090 & 0.060 & 0.057 & 0.045 & 0.051 & 0.051 & 0.052 & 0.055 & 0.047 & 0.072 & 0.075 & 0.102 \\
\hline \multirow{2}{*}{ CBF 97} & 19.18 & 18.81 & 18.64 & 18.49 & 18.29 & 18.17 & 17.83 & 17.79 & 17.72 & 17.61 & 17.53 & 17.37 & 17.44 \\
\hline & 0.125 & 0.105 & 0.091 & 0.086 & 0.067 & 0.068 & 0.063 & 0.059 & 0.069 & 0.057 & 0.065 & 0.056 & 0.094 \\
\hline \multirow[t]{2}{*}{ CBF 112} & 19.08 & 18.79 & 18.74 & 18.51 & 18.49 & 18.40 & 18.33 & 18.33 & 18.22 & 18.04 & 18.08 & 18.07 & 18.17 \\
\hline & 0.082 & 0.071 & 0.059 & 0.063 & 0.053 & 0.056 & 0.059 & 0.065 & 0.071 & 0.063 & 0.080 & 0.082 & 0.161 \\
\hline \multirow[t]{2}{*}{ CBF 118} & 18.32 & 18.00 & 17.88 & 17.66 & 17.52 & 17.39 & 17.27 & 17.13 & 17.05 & 17.10 & 16.97 & 16.72 & 16.78 \\
\hline & 0.163 & 0.154 & 0.123 & 0.126 & 0.091 & 0.094 & 0.087 & 0.084 & 0.084 & 0.084 & 0.089 & 0.066 & 0.089 \\
\hline \multirow[t]{2}{*}{ CBF 119} & 18.48 & 18.16 & 18.09 & 17.87 & 17.72 & 17.60 & 17.54 & 17.34 & 17.26 & 17.31 & 17.16 & 16.90 & 16.95 \\
\hline & 0.160 & 0.154 & 0.122 & 0.126 & 0.093 & 0.094 & 0.096 & 0.082 & 0.086 & 0.081 & 0.086 & 0.063 & 0.089 \\
\hline \multirow[t]{2}{*}{ CBF 130} & 17.67 & 17.32 & 17.48 & 17.22 & 17.43 & 17.06 & 16.85 & 16.81 & 16.78 & 17.13 & 16.66 & 16.68 & 16.60 \\
\hline & 0.089 & 0.078 & 0.079 & 0.089 & 0.087 & 0.080 & 0.066 & 0.075 & 0.086 & 0.114 & 0.087 & 0.093 & 0.108 \\
\hline \multirow[t]{2}{*}{ CBF 131} & 18.27 & 17.87 & 17.87 & 17.75 & 17.54 & 17.48 & 17.32 & 17.32 & 17.32 & 17.34 & 17.21 & 17.24 & 17.08 \\
\hline & 0.113 & 0.089 & 0.074 & 0.079 & 0.052 & 0.054 & 0.049 & 0.052 & 0.061 & 0.058 & 0.069 & 0.077 & 0.085 \\
\hline \multirow[t]{2}{*}{ CBF 161} & 19.364 & 19.028 & 18.869 & 18.660 & 18.457 & 18.339 & 18.180 & 18.074 & 17.956 & 17.942 & 17.951 & 17.631 & 17.631 \\
\hline & 0.077 & 0.054 & 0.039 & 0.034 & 0.033 & 0.033 & 0.034 & 0.036 & 0.035 & 0.038 & 0.059 & 0.043 & 0.078 \\
\hline \multirow[t]{2}{*}{ M 5} & 19.17 & 18.70 & 18.52 & 18.28 & 18.05 & 17.94 & 17.78 & 17.63 & 17.63 & 17.53 & 17.61 & 17.38 & 17.42 \\
\hline & 0.084 & 0.051 & 0.037 & 0.043 & 0.031 & 0.034 & 0.032 & 0.036 & 0.037 & 0.035 & 0.050 & 0.038 & 0.076 \\
\hline \multirow[t]{2}{*}{ M 12} & 17.99 & 17.67 & 17.53 & 17.29 & 17.15 & 17.04 & 16.83 & 16.78 & 16.74 & 16.64 & 16.53 & 16.41 & 16.36 \\
\hline & 0.116 & 0.114 & 0.089 & 0.086 & 0.064 & 0.064 & 0.038 & 0.051 & 0.055 & 0.051 & 0.056 & 0.058 & 0.061 \\
\hline \multirow[t]{2}{*}{ M33 } & 17.06 & 16.65 & 16.52 & 16.35 & 16.16 & 16.11 & 15.97 & 15.93 & 15.87 & 15.74 & 15.69 & 15.64 & 15.53 \\
\hline & 0.045 & 0.034 & 0.026 & 0.031 & 0.022 & 0.024 & 0.022 & 0.022 & 0.023 & 0.020 & 0.022 & 0.021 & 0.024 \\
\hline \multirow[t]{2}{*}{ M 51} & 18.07 & 17.70 & 17.64 & 17.52 & 17.35 & 17.32 & 17.21 & 17.18 & 17.12 & 17.06 & 17.01 & 16.98 & 16.85 \\
\hline & 0.030 & 0.022 & 0.018 & 0.021 & 0.017 & 0.019 & 0.019 & 0.022 & 0.023 & 0.024 & 0.038 & 0.035 & 0.042 \\
\hline
\end{tabular}

${ }^{a}$ CBF identifications are from Chandar et al. (1999a, 2001); M identifications are from Mochejska et al. (1998); The others are from Christian \& Schommer (1982).

the physics of the stellar structure and in the spectral libraries. For example, Charlot et al. (1996) evaluated the uncertainties in stellar population synthesis models by analyzing in detail the origin of the discrepancies between three models (Bertelli et al. 1994; Worthey 1994, BC96), and showed the main uncertainties originate from the underlying stellar evolution theory, the color-temperature scale of giant stars, and the flux libraries. Cardiel et al. (2003) also discussed in detail the problem of disentangling stellar population properties using the spectroscopic data. Vazdekis et al. (2001) investigated the origin of the discrepancy between the spectroscopic age and the CMD age for the Milky Way GC 47 Tuc, and found that the $\alpha$-enhanced isochrones with atomic diffusion included can provide a good fit to the CMD of 47 Tuc and lead to a spectroscopic age in better agreement with the CMD age.

\section{Metallicity estimates}

\subsection{Correlation between color index and metallicity}

To study the integrated properties of the stellar population in M 81, Kong et al. (2000) used the simple stellar population synthesis models of BC96. First, they convolved the SEDs of BC96 with the BATC filter profiles to obtain the optical and near-infrared integrated luminosity. When they plot the relations between color and age, they found that, among all the BATC filter bands, the color index centered at $8510 \AA$ is much more sensitive to the metallicity than to the age (see Fig. 3 of Kong et al. 2000 for details). The center of this filter band $(8510 \AA)$ is near the CaT. A good relationship between the flux ratio of $I_{8510} \equiv L_{8510} / L_{9170}$ and the metallicity for stellar populations older than $1 \mathrm{Gyr}$ was found (Eq. (4) of Kong et al. 2000),

$Z=\left(0.83-0.84 \times I_{8510}\right)^{2}$.

\subsection{Results}

Using Eq. (7), we can calculate the metallicities of these old star clusters. We obtained $I_{8510} \equiv L_{8510} / L_{9170}$ using the photometric magnitudes in BATC13 and BATC14 bands (see Table 3). Then, the metallicities can be obtained using Eq. (7). The results are listed in Table $4\left([\mathrm{Fe} / \mathrm{H}]=\log Z-\log Z_{\odot}\right)$. The uncertainties for the metallicities are just the formal errors, since Kong et al. (2000) did not discuss any errors and uncertainties when they derived Eq. (7). The formal errors are obtained in the following way. Random values are selected for the observed data such that they obey a normal distribution, with sigma determined by the known errors in each sampled bin. We then obtain the best-fit metallicity. This procedure was repeated 300 times, giving us 300 separate determinations of the best-fit metallicity. The statistical standard deviation of metallicity from this procedure is adopted as the final error for the metallicity. In Table 4, we also present the ages for the sample clusters from Ma et al. (2001, 2002a,b,c). Table 5 lists some sample cluster metallicities from other authors (Cohen et al. 1984; Christian \& Schommer 1988; Brodie \& Huchra 1991; Sarajedini et al. 1998). Using two 
Table 4. Metallicities of 31 old star clusters in M 33.

\begin{tabular}{ccccc}
\hline \hline Cluster & $B-V$ & $V-I$ & {$[\mathrm{Fe} / \mathrm{H}]$} & log age $(\mathrm{yr})$ \\
\hline U 49 & 0.68 & 1.029 & $-1.75 \pm 0.036$ & 9.60 \\
R 12 & 1.03 & 1.154 & $-1.46 \pm 0.036$ & 10.00 \\
R 14 & 0.98 & 1.311 & $-0.63 \pm 0.036$ & 9.11 \\
M 9 & 0.69 & 1.016 & $-1.17 \pm 0.108$ & 9.63 \\
U 77 & 0.67 & 0.994 & $-0.76 \pm 0.048$ & 9.20 \\
H 38 & 0.73 & 1.070 & $-0.84 \pm 0.036$ & 9.70 \\
C 20 & 0.77 & 1.045 & $-1.30 \pm 0.072$ & 9.95 \\
C 38 & 0.73 & 0.883 & $-2.12 \pm 0.108$ & 9.28 \\
H 10 & 0.96 & 1.243 & $-0.74 \pm 0.036$ & 9.90 \\
U 137 & 0.83 & 1.099 & $-0.20 \pm 0.072$ & 10.27 \\
CBF 11 & $\ldots$ & $\ldots$ & $-1.30 \pm 0.240$ & 10.30 \\
CBF 20 & $\ldots$ & $\ldots$ & $-0.44 \pm 0.240$ & 9.54 \\
CBF22 & 0.513 & $\ldots$ & $-1.03 \pm 0.481$ & 9.26 \\
CBF 28 & 0.794 & $\ldots$ & $-1.94 \pm 0.240$ & 9.80 \\
CBF 49 & 0.824 & $\ldots$ & $-1.69 \pm 0.481$ & 9.34 \\
CBF 59 & $\ldots$ & $\ldots$ & $-0.14 \pm 0.120$ & 9.11 \\
CBF 69 & $\ldots$ & 1.061 & $-1.25 \pm 0.120$ & 9.76 \\
CBF 74 & $\ldots$ & 1.061 & $-0.12 \pm 0.120$ & 9.32 \\
CBF 77 & $\ldots$ & 0.438 & $-0.62 \pm 0.240$ & 9.01 \\
CBF 87 & $\ldots$ & 1.151 & $-0.97 \pm 0.120$ & 10.06 \\
CBF 97 & $\ldots$ & 1.015 & $-0.24 \pm 0.120$ & 10.28 \\
CBF 112 & $\ldots$ & 0.846 & $-2.98 \pm 0.240$ & 9.21 \\
CBF 118 & $\ldots$ & 0.983 & $0.13 \pm 0.120$ & 9.16 \\
CBF 119 & $\ldots$ & 0.940 & $0.17 \pm 0.120$ & 9.16 \\
CBF 130 & $\ldots$ & 0.667 & $-1.63 \pm 0.240$ & 9.06 \\
CBF 131 & $\ldots$ & 0.898 & $-1.19 \pm 0.240$ & 9.30 \\
CBF 161 & $\ldots$ & $\ldots$ & $0.32 \pm 0.120$ & 10.28 \\
M 5 & 0.73 & 1.32 & $0.06 \pm 0.120$ & 10.24 \\
M 12 & 0.66 & 1.11 & $-0.54 \pm 0.120$ & 9.63 \\
M 33 & 0.71 & 1.00 & $-1.51 \pm 0.120$ & 9.21 \\
M 51 & 0.64 & 0.65 & $-1.41 \pm 0.120$ & 9.01 \\
\hline & & & &
\end{tabular}

reddening-independent techniques, Cohen et al. (1984) obtained abundance estimates for the four GCs, M9, U 49, H38, and $\mathrm{C} 20$. Comparing the results for these four clusters, we find that the only very discrepant result is for M9, which we derive to be moderately metal rich, while Cohen et al. (1984) estimated it to be very metal poor. However, the results of Christian \& Schommer (1988) and Sarajedini et al. (1998) for M 9 are intermediate between Cohen et al. (1984) and this study. Christian \& Schommer (1988) and Brodie \& Huchra (1991) estimated the metallicities for the $10 \mathrm{GCs}$ using integrated spectra. The mean metallicity difference (the values of this paper minus the values of Christian \& Schommer 1988; Brodie \& Huchra 1991) is $\langle\Delta[\mathrm{Fe} / \mathrm{H}]\rangle=0.153 \pm 0.192$. Sarajedini et al. (1998) estimated the metallicities for these $10 \mathrm{GCs}$ based on the shape and color of the red giant branch. Our results are consistent with Sarajedini et al. (1998) except for C38, which we find is very metal poor, but Sarajedini et al. (1998) find it to be the most metal rich. The mean metallicity difference (the values of this paper minus the values of Sarajedini et al. 1998 ) is $\langle\Delta[\mathrm{Fe} / \mathrm{H}]>=0.148 \pm 0.216$. Sarajedini et al. (2000) estimated the cluster metallicities using the integrated $B-V$ colors from Christian \& Schommer (1988) and the equations of Couture et al. (1990), and presented that the metallicity of R12 is very metal rich. Except for this cluster, our results are also consistent with the ones obtained using $B-V$ colors, and the mean difference $\left(<[\mathrm{Fe} / \mathrm{H}]_{\text {This paper }}-[\mathrm{Fe} / \mathrm{H}]_{B-V}>\right)$ is $0.056 \pm 0.273$.

\section{Some properties of old star clusters}

The following statistical relations are based on our data and are thus model-dependent.

\subsection{Metallicity as a function of deprojected distance}

Vílchez et al. (1988) studied the abundance gradient in M 33 on the data of emission lines in selected HII regions. The $\mathrm{O} / \mathrm{H}$ gradient is steep in the inner regions, but much flatter in the outer regions, and $\mathrm{N} / \mathrm{O}$ is constant over most of the visible disk, but lower in the outer HII region. We can investigate the radial abundance behavior of the old clusters in M 33. Using the data of both the red and blue portions of the instability trip of two halo globular clusters (M9 and U 77) and the RR Lyrae luminosity relation, Sarajedini et al. (2000) estimated M 33 to be at a distance of $(m-M)_{0}=24.84 \pm 0.16$, which is adopted in this paper. We also adopted the inclination and position angles to be $56^{\circ}$ and $23^{\circ}$ of M 33, respectively (Regan \& Vogel 1994). When the line of intersection (i.e. the major axis of the image) between the galactic plane and tangent plane is taken as the polar axis, it is easily proved that

$r=\rho \sqrt{1+\tan ^{2} \gamma \sin ^{2} \theta}$

and

$\tan \phi=\frac{\tan \theta}{\cos \gamma}$,

where $r$ and $\phi$ are the polar co-ordinates in the galactic plane, and $\rho$ and $\theta$ are the corresponding co-ordinates in the tangent plane, and $\gamma$ is the inclination angle of the galactic disk. Using formula (8), we can obtained the distances of our sample clusters from the center of M 33. Figure 2 displays the variation of metal abundance with deprojected radial position in units of kpc for 23 globular cluster candidates in M 33. This figure presents no relationship between the metal abundance of a cluster and its distance from the galactic center. This conclusion is consistent with that in Sarajedini et al. (2000) for the 9 GCs in this galaxy. Figure 3 plots the variation of metal abundance with deprojected radial position for all old clusters in this study.

\subsection{Age as a function of deprojected distance}

In Fig. 4, we plot the relation between age and galactocentric distance for 23 "true" GCs of M 33. Here we note that no relationship exists. This conclusion is consistent with Sarajedini \& King (1989) and Chaboyer et al. (1996) for the GCs in the Galaxy. Figure 5 shows this relation for all 31 old clusters in this study, and no relationship can be found, too.

\section{Summary and discussion}

In this paper, based on the results of multicolor spectrophotometry in Ma et al. (2001, 2002a,b,c) and on the formula of 
Table 5. Comparison of metallicity estimates with previous measurements.

\begin{tabular}{cccccc}
\hline \hline Cluster & {$[\mathrm{Fe} / \mathrm{H}]^{\mathrm{a}}$} & {$[\mathrm{Fe} / \mathrm{H}]_{\mathrm{CPS}}^{\mathrm{b}}$} & {$[\mathrm{Fe} / \mathrm{H}]_{\mathrm{CS}}^{\mathrm{c}}$} & {$[\mathrm{Fe} / \mathrm{H}]_{\mathrm{BH}}^{\mathrm{d}}$} & {$[\mathrm{Fe} / \mathrm{H}]_{\mathrm{S}}^{\mathrm{e}}$} \\
\hline U 49 & $-1.75 \pm 0.036$ & -1.4 & $-0.8 \pm 0.3$ & $-1.70 \pm 0.53$ & $-1.64 \pm 0.20$ \\
R 12 & $-1.46 \pm 0.036$ & $\ldots$ & $-1.2 \pm 0.3$ & $\ldots$ & $-1.19 \pm 0.24$ \\
R 14 & $-0.63 \pm 0.036$ & $\ldots$ & $-1.5 \pm 0.3$ & $\ldots$ & $-1.00 \pm 0.50$ \\
M 9 & $-1.17 \pm 0.108$ & -2.2 & $-1.7 \pm 0.3$ & $\ldots$ & $-1.64 \pm 0.28$ \\
U 77 & $-0.76 \pm 0.048$ & $\ldots$ & $\ldots$ & $-1.77 \pm 0.77$ & $-1.56 \pm 0.30$ \\
H38 & $-0.84 \pm 0.036$ & -1.0 & $-1.5 \pm 0.3$ & $\ldots$ & $-1.10 \pm 0.10$ \\
C 20 & $-1.30 \pm 0.072$ & -1.1 & $-2.2 \pm 0.3$ & $-1.25 \pm 0.79$ & $-1.25 \pm 0.22$ \\
C 38 & $-2.12 \pm 0.108$ & $\ldots$ & $-1.2 \pm 0.3$ & $\ldots$ & $-0.65 \pm 0.16$ \\
H 10 & $-0.74 \pm 0.036$ & $\ldots$ & $\ldots$ & $-0.91 \pm 0.90$ & $-1.44 \pm 0.26$ \\
U 137 & $-0.20 \pm 0.072$ & $\ldots$ & $\ldots$ & $-0.12 \pm 0.38$ & $-0.98 \pm 0.16$ \\
\hline
\end{tabular}

a This paper.

${ }^{\mathrm{b}}$ Cohen et al. (1984).

${ }^{c}$ Christian \& Schommer (1988).

${ }^{\mathrm{d}}$ Brodie \& Huchra (1991).

e Sarajedini et al. (1998).

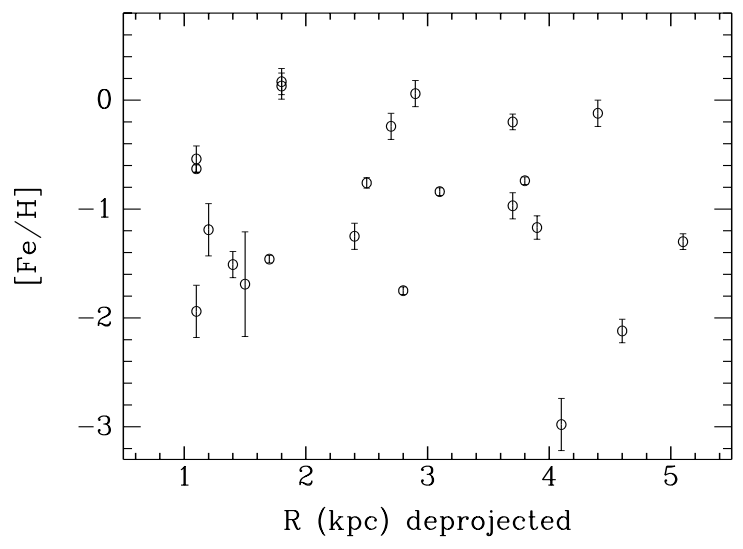

Fig. 2. Deprojected radial variation of metal abundance for 23 "true" GCs of M 33 .

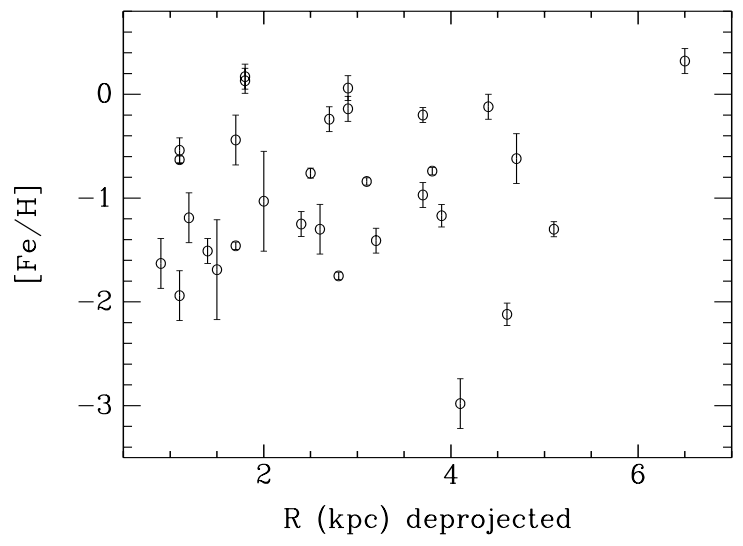

Fig. 3. Deprojected radial variation of metal abundance for 31 old clusters of M 33.

Kong et al. (2000), we estimate the metallicities of 31 old star clusters in M 33, of which there are 23 "true" GCs. The results show that most of these old clusters are metal poor. At the same time, we compare our results with others (Cohen et al. 1984; Christian \& Schommer 1988; Brodie \& Huchra 1991;

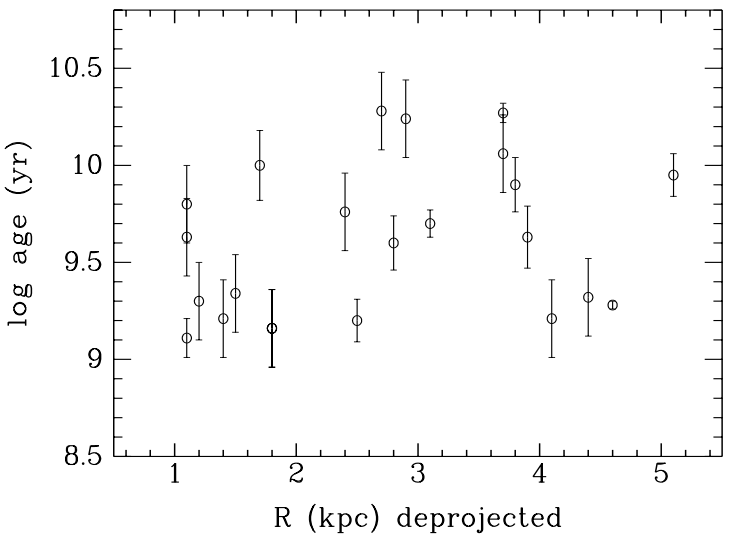

Fig. 4. Age as a function of galactocentric distance for 23 "true" GCs of M 33 .

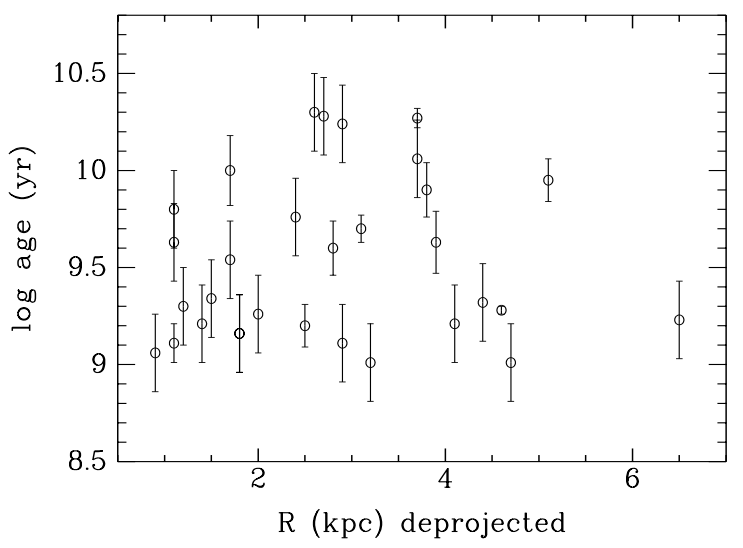

Fig. 5. Age as a function of galactocentric distance for 31 old clusters of M 33 .

Sarajedini et al. 1998) derived using different methods, such as integrated spectra and photometry. In general, our results are consistent. The statistical results show that, the ages and metal abundances based on our data do not vary with deprojected radial position. 
As we know, the old stellar populations and the nuclei of spiral galaxies are dominated by $\mathrm{G}, \mathrm{K}$ and $\mathrm{M}$ stars and therefore emit bulk of their light in the near infrared region of the spectrum. The CaT feature has been the subject of several analyses. Different authors emphasized its different utilizations, such as a luminosity indicator, and a possible discriminator between the light contribution due to dwarfs and giants in a given population mix (1997). Bica \& Alloin (1987) presented CCD spectra with $12.5 \AA$ resolution for 30 star clusters, and measured the near-infrared continuum distribution and the equivalent widths of 13 absorption features. They found that in the near-infrared spectral range, metallicity is the dominant parameter. Based on the analysis of stars (giant stars, supergiants and dwarfs), star clusters, and galaxy nuclei, Alloin \& Bica (1989) showed that the equivalent widths of $\mathrm{CaT}$ are metallicity dependent, although not as much as other metallic or molecular features ( $\mathrm{CN}$ or $\mathrm{Mg}+\mathrm{MgH})$. Armandroff \& Zinn (1988) found that the strength of the $\mathrm{CaT}$ in the integrated spectra of GCs forms a one-parameter family, this parameter being the metallicity. Erdelyi-Mendes \& Barbuy (1991) calculated synthetic spectra for the $\mathrm{Ca}$ II lines in the local thermodynamic equilibrium approximation using the model atmospheres computed by interpolation in the grids of models by Gustafsson et al. (1975) and an unpublished grid of dwarf models (see Erdelyi-Mendes \& Barbuy 1991 for details). By a detailed analysis of the behavior of the strength of $\mathrm{Ca}$ II lines as a function of stellar parameters, Erdelyi-Mendes \& Barbuy (1991) concluded that CaT has a weak dependence on the effective temperature, a modest dependence on surface gravity, but a quite important dependence on metallicity, i.e., an exponential dependence between the flux of the 2 strongest Ca II lines ( $\lambda \lambda 8542 \AA$ and $8662 \AA)$ and metallicity. Mallik (1994) presented the observations of the infrared triplet lines of ionized $\mathrm{Ca}$ for 91 stars in the spectral range F8-M4 of all luminosity classes and in the metallic range $-0.65-+0.60$, and found that the dependence of the CaT fluxes on gravity and metallicity is intricately connected, but for supergiants a strong relationship can be found. He also indicated that when a large metallicity range is considered (i.e. $\geq 1.0 \mathrm{dex}$ ), the influence of the metallicity on the CaT becomes conspicuous. Idiart et al. (1997) also confirmed the strong dependence of $\mathrm{CaT}$ index on metallicity.

Acknowledgements. We would like to thank the anonymous referee for his/her insightful comments and suggestions that improved this paper. This work has been supported by the National Key Basic Research Science Foundation (NKBRSF TG199075402) and in part by the National Science Foundation.

\section{References}

Alloin, D., \& Bica, E. 1989, A\&A, 217, 57

Armandroff, T. E., \& Zinn, R. 1988, AJ, 96, 92

Bertelli, G., Bressan, A., Chiosi, C., Fagotto, F., \& Nasi, E. 1994, A\&AS, 106, 275

Bica, E., \& Alloin, D. 1987, A\&A, 186, 49

Brodie, J., \& Huchra, J. 1991, ApJ, 379, 157

Cardiel, N., Gorgas, J., Sanchez-Blazquez, P., et al. 2003, A\&A, 409, 511
Chandar, R., Bianchi, L., \& Ford, H. C. 1999a, ApJS, 122, 431

Chandar, R., Bianchi, L., \& Ford, H. C. 1999b, ApJ, 517, 668

Chandar, R., Bianchi, L., Ford, H. C., \& Salasnich, B. 1999c, PASP, 111, 794

Chandar, R., Bianchi, L., \& Ford, H. C. 2001, A\&A, 366, 498

Chandar, R., Bianchi, L., Ford, H. C., \& Sarajedini, A. A. 2002, ApJ, 564,712

Charlot, S., Worthey, G., \& Bressan, A. 1996, ApJ, 457, 625

Christian, C. A., \& Schommer, R. A. 1982, ApJS, 49, 405

Christian, C. A., \& Schommer, R. A. 1988, AJ, 95, 704

Cohen, J. G., Persson, S. E., \& Searle, L. 1984, ApJ, 218, 141

Couture, J., Harris, W. E., \& Allwright, J. W. B. 1990, ApJS, 73, 671

Erdelyi-Mendes, M., \& Barbuy, B. 1991, A\&A, 241, 176

Fan, X., Burstein, D., Chen, J.-S., et al. 1996, AJ, 112, 628

Fukugita, M., Ichikawa, T., Gunn, J. E., et al. 1996, AJ, 111, 1748

Gustafsson, B., Bell, R. A., Eriksson, K., \& Nordlund, A. 1975, A\&A, 42, 407

Hiltner, W. A. 1960, ApJ, 131, 163

Idiart, T. P., Thévenin, F., \& de Freitas Pacheco, J. A. 1997, AJ, 113, 1066

Huchra, J., Brodie, J., Caldwell, N., Christian, C., \& Schommer, R. 1996, ApJS, 102, 29

Kaluzny, J., Stanek, K. Z., Krockenberger, M., Sasselov, D., Tonry, J. L., \& Mateo, M. 1998, AJ, 115, 1016

Kennicutt, R. C. 1998, ARA\&A, 36, 189

Kong, X., Zhou, X., Chen, J., et al. 2000, AJ, 119, 2745

Kron, G. E., \& Mayall, N. U. 1960, AJ, 65, 581

Leitherer, C., Alloin, D., Fritze-v. Alvensleben, U., et al. 1996, PASP, 108, 996

Ma, J., Zhou, X., Kong, X., et al. 2001, AJ, 122, 1796

Ma, J., Zhou, X., Chen, J., et al. 2002a, A\&A, 385, 404

Ma, J., Zhou, X., Chen, J., et al. 2002b, AJ, 123, 3141

Ma, J., Zhou, X., Chen, J., et al. 2002c, Acta Astron., 52, 453

Mallik, S. V. 1994, A\&AS, 103, 279

Melnick, J., \& D’Odorico, S. 1978, A\&AS, 34, 249

Mochejska, B. J., Kaluzny, J., Krockenberger, M., Sasselov, D. D., \& Stanek, K. Z. 1998, Acta Astron., 48, 455

Oke, J. B., \& Gunn, J. E. 1983, ApJ, 266, 713

Regan, M. W., \& Vogel, S. N. 1994, ApJ, 434, 536

Searle, L., Sargent, W. L. W., \& Bagnuolo, W. G. 1973, ApJ, 179, 427

Sarajedini, A. A., \& King, C. R. 1989, AJ, 98, 1624

Sarajedini, A. A., Geisler, D., Harding, P., \& Schommer, R. 1998, ApJ, 508, L37

Sarajedini, A. A., Geisler, D., Schommer, R., \& Harding, P. 2000, AJ, 120,2437

Schommer, R. A., Christian, C. A., Caldwell, N., Bothun, G. D., \& Huchra, J. 1991, AJ, 101, 873

Stanek, K. Z., Kaluzny, J., Krockenberger, M., et al. 1998, AJ, 115, 1894

Tinsley, B. M. 1972, A\&A, 20, 382

van den Bergh, S. 1991, PASP, 103, 609

van den Bergh, S. 1999, ARA\&A, 9, 273

Vazdekis, A., Salaris, M., Arimoto, N., \& Rose, J. A. 2001, ApJ, 549, 274

Vílchez, J. M., Pagel, B. E. J., Díaz, A. I., Terlevich, E., \& Edmunds, M. G. 1988, MNRAS, 235, 633

Worthey, G. 1994, ApJS, 95, 107

Yan, H. J., Burstein, D., Fan, X., et al. 2002, PASP, 112, 691

Zheng, Z. Y., Shang, Z., Su, H., et al. 1999, AJ, 117, 2757

Zhou, X., Jiang, Z. J., Xue, S. J., et al. 2001, ChJAA, 1, 372

Zhou, X. 1991, A\&A, 248, 367 